\title{
Intérêt des membranes en polytétrafluoroéthylène de haute densité supportant les expositions en régénération tissulaire guidée: présentation d'un cas de préservation alvéolaire.
}

\author{
Weidmann P1, Tarragano $\mathrm{H}^{1}$, Roche $\mathrm{Y}^{1}$
}

1. Pôle d'Odontologie de l'Hôpital Rothschild. (GHUEP) - Université Paris Diderot - Paris 7

\section{Introduction}

Afin de limiter la résorption alvéolaire après avulsion dentaire, des techniques spécifiques de régénération tissulaire guidée (RTG) ont été développées (Araùjo et al. J Clin Periodontol, 2005; 32(2):212). Ces techniques, dites de préservation alvéolaire, consistent en la mise en place d'une membrane associée ou non à un biomatériau de comblement. Les premières membranes utilisées étaient des membranes nonrésorbables en polytétrafluoroéthylène expansé (ePTFE). Ces membranes dites macroporeuses imposaient une fermeture muqueuse primaire afin d'éviter une infection suite à la pénétration bactérienne à travers les pores. Depuis, des membranes en polytétrafluoroéthylène de haute densité (dPTFE) microporeuses ont été mises au point afin de s'opposer à la pénétration bactérienne au sein du défaut osseux protégé tout en étant exposées au milieu oral (Carbonell et al. Int J Oral Maxillofac Surg, 2014; 43:75-84). Un cas de préservation alvéolaire par mise en place d'une membrane dPTFE exposée est ici présenté.

\section{Matériels et Méthodes}

Le cas d'un homme de 32 ans, sans antécédents médico-chirurgicaux est rapporté. Le patient présentait une mobilité 4 sur l'échelle de Mühlemann au niveau de la dent 47. Cette mobilité était associée à une douleur spontanée majorée à la percussion et à la palpation vestibulaire. A l'examen radiographique de type panoramique, une lésion radio-claire apicale était objectivée. Le diagnostic d'abcès apical aigu a alors été posé, indiquant l'avulsion de la dent 47. Une réhabilitation fixe étant envisagée après gestion de l'urgence, l'avulsion a été associée à une technique de préservation alvéolaire par la mise en place d'une membrane dPTFE laissée délibérément exposée. Aucun matériau de comblement n'a été utilisé afin de combler le défaut après l'avulsion dentaire. La membrane a été stabilisée par un appui sur les corticales résiduelles de l'alvéole puis laissée exposée au sein de la cavité orale sans traction du lambeau vestibulaire. Résultats La membrane dPTFE a été retirée après 6 semaines. La dépose, sans anesthésie, a été réalisée par simple traction. Un tissu conjonctif était retrouvé sous la membrane. Deux mois après la dépose, l'examen tomographique objectivait une quantité osseuse suffisante pour la mise en place d'implants dans ce secteur. La qualité de l'os régénéré a permis d'obtenir une stabilité primaire autorisant la mise en place de piliers de cicatrisation. Quatre mois après chirurgie, les couronnes supra-implantaires ont été réalisées.

\section{Discussion}

L'armature titane de la membrane utilisée a permis une stabilisation suffisante de cette dernière, sans biomatériaux de comblement. L'exposition de la membrane dPTFE n'a pas perturbé le processus de cicatrisation osseuse. La capacité des membranes dPTFE à tolérer l'exposition au milieu oral permet d'éviter la traction des tissus mous.

\section{Conclusion}

La régénération des tissus durs et tissus mous représente un défi permanent en implantologie. L'absence de traction tissulaire après mise en place d'une membrane dPTFE exposée non seulement réduit le temps opératoire mais permet aussi un maintien de l'architecture gingivale. La technique chirurgicale est simple et sa rapidité minimise les suites opératoires.

weidmannpierre03@gmail.com

(C) The authors, published by EDP Sciences. This is an Open Access article distributed under the terms of the Creative Commons Attribution License 4.0 (http://creativecommons.org/licenses/by/4.0/). 